\title{
The evolution of the molluscan biota of Sabaudia Lake: a matter of human history
}

\author{
ARMANDO MACALI ${ }^{1}$, ANXO CONDE $^{2,3}$, CARLO SMRIGLIO $^{1}$, PAOLO MARIOTTINI $^{1}$ \\ and FABIO CROCETTA ${ }^{4}$ \\ ${ }^{1}$ Dipartimento di Biologia, Università Roma Tre, Viale Marconi 446, I-00146 Roma, Italy. \\ ${ }^{2}$ IBB-Institute for Biotechnology and Bioengineering, Center for Biological and Chemical Engineering, \\ Instituto Superior Técnico (IST), 1049-001, Lisbon, Portugal. \\ ${ }^{3}$ Departamento de Ecoloxía e Bioloxía Animal, Universidade de Vigo, Lagoas-Marcosende, Vigo E-36310, Spain. \\ ${ }^{4}$ Stazione Zoologica Anton Dohrn, Villa Comunale, I-80121 Napoli, Italy. E-mail: fabio.crocetta@ szn.it
}

\begin{abstract}
SUMMARY: The evolution of the molluscan biota in Sabaudia Lake (Italy, central Tyrrhenian Sea) in the last century is hereby traced on the basis of bibliography, museum type materials, and field samplings carried out from April 2009 to September 2011. Biological assessments revealed clearly distinct phases, elucidating the definitive shift of this human-induced coastal lake from a freshwater to a marine-influenced lagoon ecosystem. Records of marine subfossil taxa suggest that previous accommodations to these environmental features have already occurred in the past, in agreement with historical evidence. Faunal and ecological insights are offered for its current malacofauna, and special emphasis is given to alien species. Within this framework, Mytilodonta Coen, 1936, Mytilodonta paulae Coen, 1936 and Rissoa paulae Coen in Brunelli and Cannicci, 1940 are also considered new synonyms of Mytilaster Monterosato, 1884, Mytilaster marioni (Locard, 1889) and Rissoa membranacea (J. Adams, 1800). Finally, human-driven environmental changes and cumulative anthropogenic pressures proved to be the whole driver of the constitution of the human-induced malacofauna studied, casting doubts on the correct use of the definition of "native fauna".
\end{abstract}

Keywords: Sabaudia Lake, Tyrrhenian Sea, Mollusca, alpha diversity, human-driven environmental changes.

RESUMEN: Evolución de la comunidad de moluscos del lago Sabaudia: Causas antropogénicas. - Se hizo un seguimiento de la evolución de la comunidad de moluscos en el lago Sabaudia (Italia, región central del mar Tirreno), considerando datos del siglo pasado y recurriendo a consultas bibliográficas, material tipo en museos y muestreos de campo realizados entre Abril de 2009 y Septiembre de 2011. Nuestro análisis biológico reveló la existencia de fases bien diferenciadas que muestran de modo claro el cambio definitivo de esta laguna inicialmente de aguas dulces que posteriormente se transformó en un ecosistema de influencia marina debido a la acción humana. El registro de taxones de subfósiles marinos sugiere un contexto ambiental similar en una época anterior, en concordancia con datos históricos. Este trabajo proporciona detalles faunísticos y ecológicos de la malacofauna del lago Sabaudia, dando especial importancia a las especies invasoras. Dentro de este amplio contexto Mytilodonta Coen, 1936, Mytilodonta paulae Coen, 1936 y Rissoa paulae Coen en Brunelli y Cannicci, 1940 son considerados nuevos sinónimos de Mytilaster Monterosato, 1884, Mytilaster marioni (Locard, 1889) y Rissoa membranacea (J. Adams, 1800). Por último, los cambios ambientales de génesis humana y la presión antropogénica a lo largo del tiempo determinaron la composición de la malacofauna estudiada, levantando dudas sobre el uso correcto del concepto "fauna nativa".

Palabras clave: lago Sabaudia, mar Tirreno, moluscos, diversidad alfa, presión antropogénica.

\section{INTRODUCTION}

Worldwide confined coastal environments have a long history as human-dominated areas (e.g. Mannino and Thomas 2002, Davenport and Davenport 2006). They also play a key role within the homogenization of the global biota, being hotspots for the introduction and secondary spreading of alien species (e.g. Occhipinti- 
Ambrogi 2007). Anthropogenic pressures, land development and eutrophication are the main causes of both the alteration of these ecosystems and the depletion of coastal resources (Beatley 1991, Nixon 1995, Lloret and Riera 2008, Claudet and Fraschetti 2010, Coll et al. 2011). Therefore, periodical annotated check lists would be a useful basis for further studies, assessments and conservation programmes, and a valid tool for characterizing different biotopes inside the study area and making long-term comparisons (Hendrickx and Harvey 1999, Mikkelsen and Cracraft 2001, Costello et al. 2006).

Lack of historical data and the absence of specialists involved in the early studies, however, often make it difficult to reconstruct possible changes occurring within an area. Sabaudia Lake (eastern-central Tyrrhenian Sea, Latium, Italy) is an evident exception to this statement. This basin had very few brackish water periods during the Roman Empire and again around 1700 . They were strictly related to the building and the re-opening of the "Roman Channel", a complex sea-lake connection $800 \mathrm{~m}$ long, located at the extreme southern tip of the basin and branched in the middle to give two secondary channels. The lack of maintenance, however, led to the closing of the Roman Channel soon after its opening, thus bringing the lake back to its original freshwater status. During the Agro Pontine environmental reclamation (around 1930), another sea-lake connection $500 \mathrm{~m}$ long, located at the northern tip of the basin and called "Caterattino", was opened. This connection is now alternatively subjected to severe hypoxia and anoxia events, and sea water flows through it only a few times per year. Sea water renewal and exchange is mainly guaranteed through the definitive re-opening of the Roman Channel. Over the last few decades the area has been subjected to several anthropogenic impacts, including sewages discharges, disturbance by recreational and professional fisheries, seasonal tourism, boat impact and mariculture (including shellfish harvesting). The Sabaudia urban area is located all along the northern tip.

Past datasets covering the local biota are quite satisfactory, and the continuous human commercial activities in the area strongly contributed to the set-up of a conspicuous biological database. Moreover, field research carried out from 1933 to 1995 also offered a quite complete overview of the local molluscan biodiversity (Brunelli and Cannicci 1934, 1940, 1944, Coen 1936, Ferrero 1961, Bini 1983a, 1987, Perdicaro 1984, Mancini et al. 1988, Di Marco et al. 1990, Alia and Russo 2003). A long-term evaluation of faunal changes and human impacts on the local faunal assemblages, however, is still lacking, mainly because of the absence of recent research. The aim of the present work is to fill this gap, through tests on whether temporal variations or qualitative modifications have occurred within the molluscan fauna of Sabaudia Lake during the last century.

\section{MATERIALS AND METHODS}

\section{Study area}

Sabaudia Lake (also known as Paola Lake) (from $41^{\circ} 18^{\prime} 19^{\prime \prime} \mathrm{N}, 013^{\circ} 01^{\prime} 09^{\prime \prime} \mathrm{E}$ to $41^{\circ} 15^{\prime} 00^{\prime} \mathrm{N}$, $013^{\circ} 02^{\prime} 40^{\prime \prime} \mathrm{E}$ ) is the largest of the four coastal lakes integrated in the Circeo National Park (CNP, Latium, Italy). It is located on the extreme southern edge of the Pontine Plain, along the central Tyrrhenian Sea coastline (Fig. 1A), and its main axis stretches along the Tyrrhenian shores for $6.7 \mathrm{~km}$, with six arms crossing it transversely, starting from the mountain side and allowing moderate freshwater to flow through (from north to south: Annunziata, Crapara, Arciglioni, Carnarola, Molella and Bagnara: Fig. 1B). It has an average depth of around 4-4.5 $\mathrm{m}$, a maximum of about $10 \mathrm{~m}$ in its southern part and a surface area of approximately 400 ha (Ferrero 1961, Perdicaro 1983, 1984). The Caterattino and the Roman Channel are figured in Figure 1B and 1C, respectively.

\section{Past composition of the molluscan assemblages}

An extensive literature survey was carried out. Indexed papers were found and examined, but grey literature was also taken into account. The latter includes historical Italian journals that are no longer published (many of which were never indexed) and malacological papers that still appear in non-indexed journals, thus only allowing for manual searching. Literature record listing was as exhaustive as possible, but for some species multiple records seem to be based on the same samples. Bibliographic data were taxonomically adjusted to allow for some comparison. Although differences in collection methods are unavoidable, and taxonomical knowledge is continuously increasing, most of the typical lagoon species are macrobenthic taxa that have been clearly recognized by biologists and malacologists for centuries, thus suggesting that their presence/absence could not be passed over unnoticed.

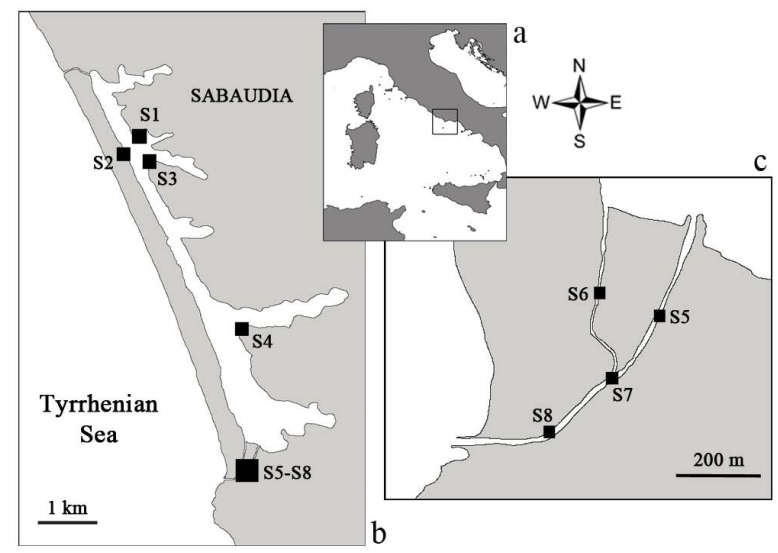

Fig. 1. Study area. A. Location of Sabaudia Lake (Italy, Tyrrhenian Sea) in the Mediterranean Sea. B-C. Sabaudia Lake. Map of the sampling sites: numbers as in Table 1. 
TABLE 1. - Sampling sites (Ss; see also Fig. 1B, C): coordinates, substrate/environmental features, dominant macrofauna (molluscs excluded), depth ranges (in metres) and sampling equipment (or techniques) used [air-lift sampler, ALS; hand dredge, HD; hand grab, HG; surface brushing, SB; hand picking, HP].

\begin{tabular}{|c|c|c|c|c|c|}
\hline Ss & Coordinates & Substrate/Environmental features & Dominant macrofauna & Depth & Samplings \\
\hline S1 & $\begin{array}{l}41^{\circ} 17^{\prime} 44^{\prime \prime} \mathrm{N}, \\
013^{\circ} 01^{\prime} 09^{\prime \prime} \mathrm{E}\end{array}$ & $\begin{array}{l}\text { Riparian vegetation at the tidal level. Sandy } \\
\text { bottom with scarce bivalves loose valvae. } \\
\text { Algal film. The oxic-anoxic horizon is at } 15- \\
20 \mathrm{~cm} \text { depth from the sediment surface. Low } \\
\text { hydrodynamism. }\end{array}$ & $\begin{array}{l}\text { Mostly composed of paralic species: live } \\
\text { and dead balanids and Ficopomatus colonies } \\
\text { on loose valvae, forming a biogenic hard } \\
\text { substrate for the settlement of Paranemonia } \\
\text { cinerea and the tunicates (Styela plicata, } \\
\text { Ciona intestinalis sp. A and Phallusia } \\
\text { mamillata) turnover. Isopoda, Anfipoda } \\
\text { (mainly gammarids and caprellids) and } \\
\text { Asterina gibbosa } \text { are present. }\end{array}$ & $0-1$ & ALS, HD, HP \\
\hline
\end{tabular}
including tree trunks and branches supplied from the riparian vegetation, often with algal film. The oxic-anoxic horizon is at $15-20$ $\mathrm{cm}$ depth from the sediment surface. Low hydrodynamism.

$41^{\circ} 17^{\prime} 35^{\prime}$ 'N, Riparian vegetation at the tidal level. 01301'13',E Many medium-sized pebbles (up to $20 \mathrm{~cm}$ diameter), up to the oxic-anoxic horizon (at $15-20 \mathrm{~cm}$ depth), on a sandy matrix. Algal film. Few small sandy patches. Low hydrodynamism.

$41^{\circ} 16^{\prime} 08^{\prime \prime}$ N, Riparian vegetation at the tidal level. 01302'14' 'E Roman bricks (from an ancient ruin) on a sandy bottom, abundant algal film and Chlorophyta. The oxic-anoxic horizon is at $15-20 \mathrm{~cm}$ depth from the sediment surface. Low hydrodynamism.

$41^{\circ} 15^{\prime} 00^{\prime}$ 'N, Mostly sandy, with deposits of shells, gravels $013^{\circ} 02^{\prime} 20^{\prime} \mathrm{E}$ and medium-sized rocks, often with algal film. A small mixed meadow composed of the seagrasses Cymodocea nodosa and Zoostera grows near to the lake entrance. Organic remains, mostly composed of shells and dead polychaetes colonies, lie along the hard artificial banks. Local water properties (such as salinity and turbidity) may rapidly change according to the tidal phase.

$41^{\circ} 15^{\prime} 02^{\prime}$ 'N, Fine sand rich in mud and organic deposits $013^{\circ} 02$ '21' $\mathrm{E}$ among the hard artificial banks. Algal film. A wide Ulva cfr. lactuca cover usually appears during spring and summer seasons. Tidal flow weaker than S5.

$41^{\circ} 14^{\prime} 54^{\prime \prime}$ N, Mostly sandy, with deposits of shells, gravels $013^{\circ} 02^{\prime} 21^{\prime \prime} \mathrm{E}$ and medium-sized rocks among the hard artificial banks. Algal film and massive deposit of organic remains, mainly compose of mouldy Posidonia oceanica and Codium cfr. vermilara leaves. The higher velocity of water flow affects the bottom particle size, which is greater. The tidal flow velocity shares its features with S5.
Higher biomasses (than at $\mathrm{S} 1$ ) of balanids and Ficopomatus settled on organic remains. Dominant macrofauna strongly resembling

that listed in S1.

0-1 HG, HD, SB, HP.

Higher biomasses (than at S1) of balanids and Ficopomatus settled on medium-sized pebbles. Dominant macrofauna strongly resembling that listed in $\mathrm{S} 1$.

Higher biomasses (than at S1) of balanids and Ficopomatus settled on Roman bricks. Dominant macrofauna strongly resembling that listed in S1.

Somehow influenced by the lagoon domain and resembling that of S1-S4, although the main contribution to the biotic assemblage is due to tunicate thalassoid species such as Clavelina, Botryllus schosseri and Botrylloides leachii. Few sponge species are present.

Resembling S5, suggesting again that its environmental features are lagoon-related. However, a wide population of Cerianthus membranaceus occurs, as well as hydroids (Aglaopheniidae and Eudendrium).

Strongly resembling S5, but with higher presence of thalassoid species and sponges. Many hydroids (Aglaopheniidae and Eudendrium).
0-1 ALS, HG, SB, HP. 0-1.5 ALS, HG,
HD, SB, HP. HD, SB, HP.

0-3.5 ALS, HG, HD, SB, HP.

0-2 ALS, HG, HD, SB, HP.
0-2.5 ALS, HG,
Paralic and thalassoid filter feeder taxa occur, although scarce. Aiptasia mutabilis and Anemonia viridis share hard substrates with few Paranemonia cinerea. Many Tubularia and Pycnogonids. Few sponge species.

\section{Current composition of the molluscan assemblage: sampling design and laboratory work}

To describe the current molluscan assemblage of Sabaudia Lake and to evaluate the faunal changes that have occurred within, the taxonomic composition was the main target. Preliminary field observations carried out since April 2007 led us to choose eight sampling sites (Table 1; Fig. 1B, C) on the basis of environmental features (substrate, dominant macrofauna and depth 


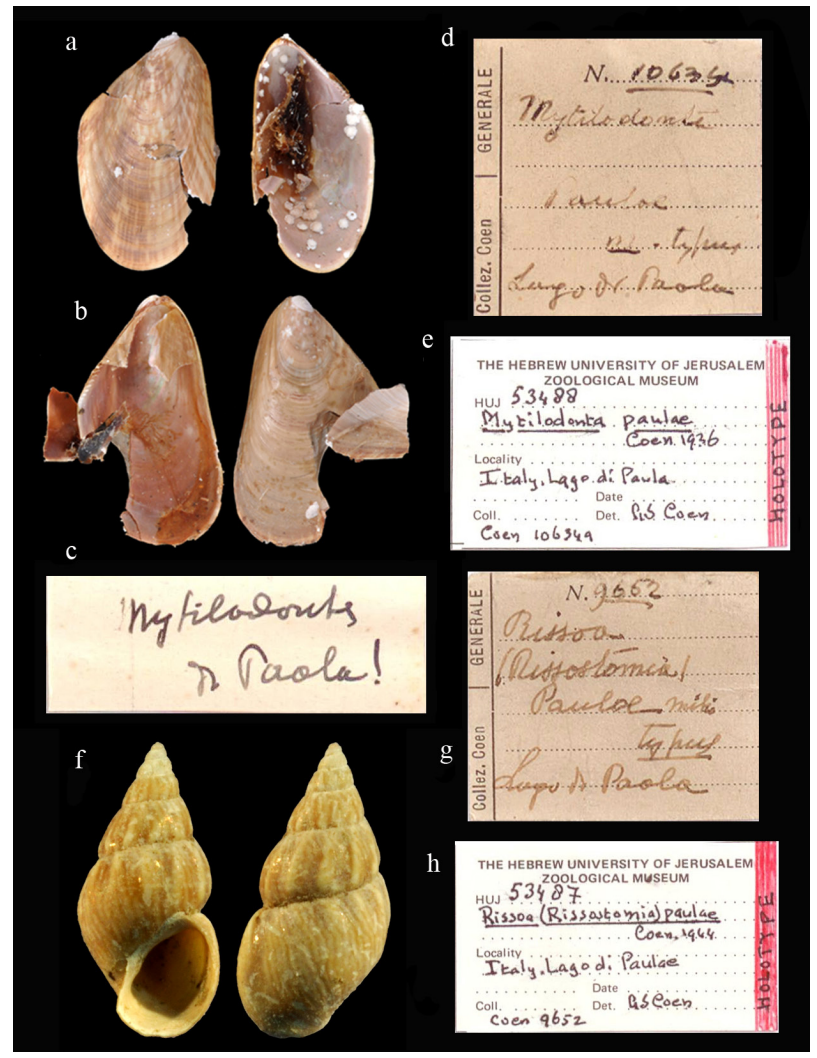

Fig. 2. Taxa described with Sabaudia Lake as type locality (not to scale, sizes reported as maximum height $\times$ maximum width) and corresponding labels. A-E. Mytilodonta Coen, 1936 and Mytilodonta paulae Coen, 1936. A, Holotype (Coen 10634a - HUJ53488) - $7.39 \times 5.22$ mm; B, Paratype (Coen 10634b - HUJ53489) - 8.72 $\mathrm{mm}$; C-D, Coen's original handwritten labels; E, Holotype label; F-H, Rissoa paulae Coen in Brunelli and Cannicci, 1940; F, Holotype (Coen 9652 - HUJ53487) - $5.09 \times 2.42 \mathrm{~mm}$; G, Coen's original handwritten label; H. Holotype label.

range), and levels of marine influence, and thereafter to approximately encompass all the different habitats present in the study area. Periodical fluctuations in abundance within the sampling sites were ignored, as was seasonal regression or spreading of taxa distributions (mostly happening within the Bagnara arm area, which was not sampled). Sampling was carried out monthly during daylight hours by two of the authors (A. Macali and P. Mariottini) from April 2009 to September 2011, by means of snorkelling and SCUBA diving. Different sampling techniques (air-lift sampler, hand grab, hand dredge, surface brushing and hand picking) were used according to the site environmental features, covering most of the micro niches available (Table 1). Sediments were sieved through a 1-mm mesh and most of the largest or abundant molluscs were promptly released after field identification, so as to minimize the impact on the local biota. Finally, special emphasis was given to alien species.

Upon arrival at the laboratory, samples were first sorted and then transferred to $98 \% \mathrm{EtOH}$ for subsequent molecular analysis. They are currently preserved at the Dipartimento di Biologia, Università Roma 3,
Rome, Italy. Identifications were mostly performed on live material and up to species level.

\section{Updated taxonomy and nomenclature}

Updated taxonomy and nomenclature used herein follow the World Register of Marine Species (last accessed 10 July 2013), with few exceptions [taxa listed as a still unsolved complex, if current knowledge does not allow taxa to be correctly identified from the morphology/anatomy only, and new synonymies proposed (see Appendix 1)]. The authorities of molluscan species listed below are mostly reported in Tables and Appendix only.

\section{Data analysis}

Sampling design was more "faunistic" than "ecological". Nevertheless, the material obtained allowed a first analysis to be made on some ecological traits of the local biota because of the exhaustive census of the molluscan species at the sampling sites. The subsequent analyses were therefore conducted on presenceabsence data only.

Non-metric multidimensional scaling was applied to the data based on the Jaccard index (Legendre and Legendre 1998). Groups of minimum interpretable sites were formed by choosing an arbitrary threshold distance in cluster analysis (Clarke and Warwick 1994), using the complete linkage agglomerative clustering (Borcard et al. 2011). Sites were grouped spatially in accordance with the similarity of species composition (Legendre and Legendre 1998).

Non-parametric permutational multivariate analysis of variance (function Adonis: Oksanen et al. 2006) was applied to test for any changes in the macrobenthic assemblages caused by the different species composition at different sites (Anderson 2001). The analysis was carried out on the dissimilarity matrix calculated using the Jaccard index. Differences in beta diversity were assessed by a test on multivariate dispersion between sites (Anderson 2001, Anderson et al. 2006). Differences in the mean number of species and mean number of shared species between sites were tested by the sign test and Wilcoxon's signed-rank test, respectively (Crawley 2007).

Values are shown as means \pm standard deviations. All analyses were carried out using the vegan package (Oksanen et al. 2006), which is run in the free $\mathrm{R}$ environment for statistical computing (R Development Core Team 2009).

\section{RESULTS}

Literature analysis revealed records of 36 live collected and subfossil taxa from Sabaudia Lake, as published from 1934 to 2011. An updated literature record listing is presented in Table 2 and full details are reported in Appendix 1. Original data from field samplings censused 118 taxa during 2005-2011, all reported in Table 3 . The ordination of the sampled sites, showing two dis- 
TABLE 2. - Records of molluscan species from the Sabaudia Lake (from the literature only). A record is marked with an asterisk (*) if a local introduction or translocation has been traced in the literature: see Appendix 1 for further details. Bold: alien species in the Italian territorial seawaters (Crocetta 2012, Crocetta et al. 2013, see Appendix 1 for discussion on C. angulata).

\begin{tabular}{|c|c|c|c|c|}
\hline Taxa & Subfossil & $1933-1958$ & 1966-1995 & 2005-2011 \\
\hline \multicolumn{5}{|l|}{ GASTROPODA } \\
\hline Gibbula adansonii (Payraudeau, 1826) complex & $\mathrm{X}$ & & & \\
\hline Bittium reticulatum (da Costa, 1778) & $\mathrm{X}$ & $\mathrm{X}$ & $\mathrm{X}$ & \\
\hline Cerithium sp. & $\mathrm{X}$ & & & \\
\hline Cerithium spp. vulgatum Bruguière, 1792 complex & $\mathrm{X}$ & & & \\
\hline Heleobia stagnorum $(\mathrm{Gmelin}, 1791)$ & & & $\mathrm{X}$ & \\
\hline Pusillina cf. lineolata (Michaud, 1832) & $\mathrm{X}$ & & & \\
\hline Rissoa membranacea (Adams J., 1800) & & $\mathrm{X}$ & & \\
\hline Ecrobia ventrosa (Montagu, 1803) & & & $\mathrm{X}$ & \\
\hline Hydrobia acuta (Draparnaud, 1805) & & $\mathrm{X}$ & $\mathrm{X}$ & \\
\hline Hydrobia sp. & & $\mathrm{X}$ & & \\
\hline Bolinus brandaris (Linnaeus, 1758) & $\mathrm{X}$ & & & \\
\hline Hexaplex trunculus (Linnaeus, 1758) & $\mathrm{X}$ & & & \\
\hline Rapana venosa (Valenciennes, 1846) & & & & $\mathrm{X}$ \\
\hline Cyclope neritea (Linnaeus, 1758) & $\mathrm{X}$ & & $\mathrm{X}$ & \\
\hline Nassarius spp. corniculum (Olivi, 1792) complex & $\mathrm{X}$ & & $\mathrm{X}$ & \\
\hline Nassarius nitidus (Jeffreys, 1867) & $\mathrm{X}$ & & & \\
\hline Bulla striata Bruguière, 1792 & $\mathrm{X}$ & & & \\
\hline Haminoea navicula (da Costa, 1778) & & $\mathrm{X}$ & & \\
\hline Haminoea orbignyana (Férussac, 1822) & & & $\mathrm{X}$ & \\
\hline \multicolumn{5}{|l|}{ BIVALVIA } \\
\hline Arcuatula senhousia (Benson in Cantor, 1842) & & & & $\mathrm{X}$ \\
\hline Mytilaster marioni (Locard, 1889) & & $\mathrm{X}$ & $\mathrm{X}$ & \\
\hline Mytilaster minimus (Poli, 1795) & & $\mathrm{X}$ & $\mathrm{X}$ & \\
\hline Mytilus galloprovincialis Lamarck, 1819 & $\mathrm{X}$ & $\mathrm{X}$ & $* \mathrm{X}$ & \\
\hline Crassostrea angulata (Lamarck, 1819) & & & $* \mathrm{X}$ & \\
\hline Crassostrea gigas (Thunberg, 1793) & & & $* \mathrm{X}$ & \\
\hline Ostrea edulis Linnaeus, 1758 & & & $* \mathrm{X}$ & \\
\hline Loripes lucinalis (Lamarck, 1818) & $\mathrm{X}$ & $\mathrm{X}$ & $\mathrm{X}$ & \\
\hline Cerastoderma spp. glaucum (Bruguière, 1789) complex & $\mathrm{X}$ & $\mathrm{X}$ & $\mathrm{X}$ & $\mathrm{X}$ \\
\hline Parvicardium exiguum (Gmelin, 1791) & & & $\mathrm{X}$ & \\
\hline Angulus tenuis (da Costa, 1778$)$ & & & $\mathrm{X}$ & \\
\hline Gastrana fragilis (Linnaeus, 1758) & & & $\mathrm{X}$ & \\
\hline Abra segmentum (Récluz, 1843) & & $\mathrm{X}$ & $\mathrm{X}$ & \\
\hline Polititapes aureus (Gmelin, 1791) & $\mathrm{X}$ & & $\mathrm{X}$ & \\
\hline Venerupis decussata (Linnaeus, 1758) & $\mathrm{X}$ & & $* \mathrm{X}$ & \\
\hline Venerupis philippinarum (Adams A. and Reeve, 1850) & & & $* \mathrm{X}$ & \\
\hline \multicolumn{5}{|l|}{ CEPHALOPODA } \\
\hline Sepia officinalis Linnaeus, 1758 & & & $* \mathrm{X}$ & \\
\hline
\end{tabular}

tinct groups identified by the cluster analysis, is reported in Figure 3. The distance from the group centroid of the channel and the inner cluster of sites is reported in Figure 4 , while the most common species observed in both clusters are reported in Table 4. Finally, faunal data as obtained were divided into four main periods (subfossil records, 1933-1958, 1966-1995, 2005-2011) so as to trace possible evolutionary states of the local molluscan biota.

\section{Subfossil records}

Subfossil records of 16 taxa were traced in the literature (Ferrero 1961, Bini 1987), including both marine and brackish water species (e.g. Bolinus brandaris, Hexaplex trunculus, Nassaridae, Cerastoderma and Veneridae: Table 2, Appendix 1).

\section{The 1933-1958 period}

Eleven taxa had been recorded by past authors (Table 2, Appendix 1). Among them, one new genus (Bivalvia: Mytilidae) and two new species (Bivalvia:

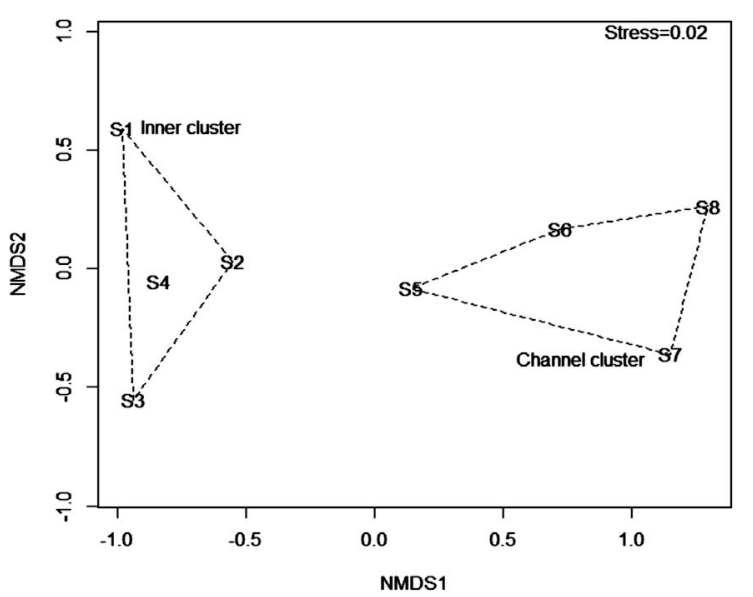

Fig. 3. Non-metric multidimensional scaling plot showing the ordination of the sites as well as the groups identified by cluster analysis: Inner and Channel Clusters.

Mytilidae and Gastropoda: Rissoidae) were described with Sabaudia Lake as the type locality (namely: Mytilodonta Coen, 1936, Mytilodonta paulae Coen, 
TABLE 3. - Molluscan species from Sabaudia Lake (present study) and their occurrence at the study sites (S) (Fig. 1B, C; Table 1). Bold: alien species in the Italian territorial seawaters (Crocetta 2012, Crocetta et al. 2013); Literature (L): X indicate species previously cited from the area on the basis of literature records. See Table 2 and Appendix 1 for further details.

\begin{tabular}{|c|c|c|c|c|c|c|c|c|c|c|}
\hline \multirow{2}{*}{$\begin{array}{l}\text { Classes } \\
\text { Family }\end{array}$} & \multirow{2}{*}{$\mathrm{L}$} & \multirow{2}{*}{ Taxa } & \multicolumn{8}{|c|}{ Sites $(\mathrm{S})$} \\
\hline & & & 1 & 2 & 3 & 4 & 5 & 6 & 7 & 8 \\
\hline \multicolumn{11}{|l|}{ POLYPLACOPHORA } \\
\hline LEPTOCHITONIDAE & & Lepidopleurus cajetanus (Poli, 1791) & & & & & & & + & + \\
\hline LEPIDOCHITONIDAE & & Lepidochitona cinerea (Linnaeus, 1767) & & & & & & & + & + \\
\hline CHITONIDAE & & Chiton olivaceus Spengler, 1797 & & & & & + & + & + & + \\
\hline $\begin{array}{l}\text { ACANTHOCHITONIDAE } \\
\text { GASTROPODA }\end{array}$ & & Acanthochitona fascicularis (Linnaeus, 1767) & & & & & & & & + \\
\hline \multirow{3}{*}{ Patellidae } & & Patella caerulea Linnaeus, 1758 & & & & & & & + & + \\
\hline & & $\begin{array}{l}\text { Patella spp. rustica (Linnaeus, 1758) complex } \\
\quad \text { (see Sà-Pinto } \text { et al. 2010) }\end{array}$ & & & & & & & $\begin{array}{l}+ \\
+\end{array}$ & $\begin{array}{l}+ \\
+\end{array}$ \\
\hline & & Patella ulyssiponensis Gmelin, 1791 & & & & & + & + & + & + \\
\hline LOTTIDAE & & Tectura virginea (Müller O.F., 1776) & & & + & + & & & & \\
\hline \multirow[t]{2}{*}{ FISSURELLIDAE } & & Diodora gibberula (Lamarck, 1822) & & & & & & + & + & + \\
\hline & & Diodora graeca Linnaeus, 1758 & & & & & & + & + & \\
\hline \multirow[t]{4}{*}{ TROCHIDAE } & $\mathrm{x}$ & $\begin{array}{l}\text { Gibbula adansonii (Payraudeau, 1826) complex } \\
\text { (see Barco et al. 2013) }\end{array}$ & & & & & + & + & + & \\
\hline & & Gibbula adriatica (Philippi, 1844) & + & + & + & + & + & + & + & + \\
\hline & & Gibbula albida (Gmelin, 1791) & + & + & + & + & + & + & + & \\
\hline & & Gibbula ardens (Von Salis, 1793) & + & + & + & + & + & + & + & \\
\hline \multirow[t]{2}{*}{ CERIthidae } & $\mathrm{X}$ & Bittium reticulatum (da Costa, 1778) & & + & + & + & + & & + & \\
\hline & $\mathrm{X}$ & $\begin{array}{l}\text { Cerithium spp. vulgatum Bruguière, } 1792 \text { complex } \\
\text { (see Bosselier-Dubayle and Gofas 1999) }\end{array}$ & & & & & + & & & \\
\hline \multirow[t]{5}{*}{ RISSOIDAE } & & Alvania cimex (Linnaeus, 1758 ) & & & & & & + & + & \\
\hline & & Alvania lineata Risso, 1826 & & & & & & + & + & \\
\hline & & Alvania mamillata Risso, 1826 & & & & & & + & + & \\
\hline & & Pusillina lineolata (Michaud, 1832) & & + & + & + & + & & & \\
\hline & & Pusillina marginata (Michaud, 1832) & & + & + & + & + & & & \\
\hline ASSIMINEIDAE & & Paludinella globularis (Hanley in Thorpe, 1844) & & & + & + & & & & \\
\hline HYDROBIIDAE & $\mathrm{X}$ & Hydrobia acuta (Draparnaud, 1805) & & & + & + & & & & \\
\hline & & Islamia pusilla (Piersanti, 1952) & & & + & + & & & & \\
\hline CALYPTRAEIDAE & & Crepidula fornicata (Linnaeus, 1758) & & + & + & + & + & & & \\
\hline MuRICIDAE & $\mathrm{X}$ & Bolinus brandaris (Linnaeus, 1758) & & & & & + & + & + & + \\
\hline & $\mathrm{X}$ & Hexaplex trunculus (Linnaeus, 1758) & + & + & + & + & + & + & + & + \\
\hline & & Muricopsis cristata (Brocchi, 1814) & & & & & & & + & + \\
\hline & & $\begin{array}{l}\text { Ocinebrina edwardsii (Payraudeau, 1826) complex } \\
\text { (see Barco et al. in press) }\end{array}$ & & & & & & & + & \\
\hline & & Ocenebra erinaceus (Linnaeus, 1758) & & & & & & + & + & + \\
\hline & & Stramonita haemastoma (Linnaeus, 1767) & + & + & + & + & + & + & + & + \\
\hline BUCCINIDAE & & Euthria cornea (Linnaeus, 1758) & & & & & + & + & + & + \\
\hline & & Pollia dorbignyi (Payraudeau, 1826) & & & & & + & & + & + \\
\hline NASSARIDAE & $\mathrm{X}$ & Cyclope neritea (Linnaeus, 1758) & + & + & + & + & + & + & & \\
\hline & $\mathrm{X}$ & $\begin{array}{l}\text { Nassarius spp. corniculus (Olivi, 1792) complex } \\
\text { (see Iannotta et al. 2009) }\end{array}$ & + & + & + & + & + & + & + & + \\
\hline & & Nassarius cuvierii (Payraudeau, 1826) & & & & & & + & + & + \\
\hline & & Nassarius incrassatus (Strøm, 1768) & + & + & + & + & + & & & \\
\hline & & Nassarius mutabilis (Linnaeus, 1758) & & & & & & & & + \\
\hline & $\mathrm{X}$ & Nassarius nitidus (Jeffreys, 1867) & + & + & + & + & + & + & + & + \\
\hline BULLIDAE & $\mathrm{X}$ & Bulla striata Bruguière, 1972 & & + & + & + & & & & + \\
\hline HAMINOEIDAE & & Haminoea japonica Pilsbry, 1895 & & + & + & + & + & & & \\
\hline AgLAJIDAE & & Aglaja tricolorata Renier, 1807 & & & & & + & & & \\
\hline PleUROBRANCHIDAE & & Berthellina edwardsi (Vayssière, 1896) & & & & & + & & + & \\
\hline & & Pleurobranchaea meckeli (Blainville, 1825) & & & & & & + & & \\
\hline APLYSIIDAE & & Aplysia depilans Gmelin, 1791 & & & & & + & + & & \\
\hline & & Aplysia fasciata Poiret, 1789 & & & & & + & + & & \\
\hline & & Aplysia punctata (Cuvier, 1803) & & & & & + & + & + & \\
\hline & & Bursatella leachii Blainville, 1817 & & & & & + & + & & \\
\hline DORIDIDAE & & Doris ocelligera (Bergh, 1881) & & & & & & & + & \\
\hline DISCODORIDIDAE & & Baptodoris cinnabarina Bergh, 1884 & & & & & & & + & \\
\hline & & Jorunna tomentosa (Cuvier, 1804) & & & & & & + & + & \\
\hline & & Peltodoris atromaculata Bergh, 1880 & & & & & & & + & \\
\hline & & Taringa armata Swennen, 1961 & & & & & & & + & \\
\hline DENDRODORIDIDAE & & Dendrodoris grandiflora (Rapp, 1827) & & & & & & & + & + \\
\hline POLYCERIDAE & & Polycera quadrilineata (Müller O.F., 1776) & & & & & & & + & \\
\hline PROTOCTONOTIDAE & & Janolus cristatus (delle Chiaje, 1841) & & & & & & + & & \\
\hline AEOLIDIIDAE & & Anteaeolidiella lurana (Marcus and Marcus, 1967) & & & & & & + & + & \\
\hline & & Spurilla neapolitana (delle Chiaje, 1841) & & & & & + & + & + & + \\
\hline FACELINIDAE & & Cratena peregrina (Gmelin, 1791) & & & & & & + & + & \\
\hline & & Godiva quadricolor (Barnard, 1927) & & & & & + & + & + & + \\
\hline FLABELLINIDAE & & Calmella cavolini (Vérany, 1846) & & & & & & + & + & + \\
\hline & & Flabellina affinis (Gmelin, 1791) & & & & & & + & + & \\
\hline & & Flabellina ischitana Hirano and Thompson, 1990 & & & & & & + & + & \\
\hline
\end{tabular}




\begin{tabular}{|c|c|c|c|c|c|c|c|c|c|c|}
\hline \multirow{2}{*}{$\begin{array}{c}\text { Classes } \\
\text { Family }\end{array}$} & \multirow{2}{*}{$\mathrm{L}$} & \multirow{2}{*}{ Taxa } & \multicolumn{8}{|c|}{ Sites $(\mathrm{S})$} \\
\hline & & & 1 & 2 & 3 & 4 & 5 & 6 & 7 & 8 \\
\hline & & Flabellina pedata (Montagu, 1815) & & & & & & + & + & \\
\hline ELLOBIIDAE & & Myosotella myosotis (Draparnaud, 1801) & + & + & + & + & & & & \\
\hline \multicolumn{11}{|c|}{ 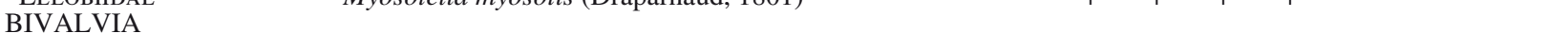 } \\
\hline Nuculidae & & Nucula nitidosa Winckworth, 1930 & & & & & + & & & \\
\hline \multirow[t]{2}{*}{ ARCIDAE } & & Arca noae Linnaeus, 1758 & & & & & + & + & + & + \\
\hline & & Barbatia barbata (Linnaeus, 1758) & & & & & & + & + & + \\
\hline NoEtiIDAe & & Striarca lactea (Linnaeus, 1758) & & & & & + & + & + & + \\
\hline \multirow[t]{5}{*}{ Mytilidae } & $\mathrm{x}$ & Arcuatula senhousia (Benson in Cantor, 1842) & & & & & & & & + \\
\hline & $\mathrm{x}$ & Mytilaster marioni (Locard, 1889) & & + & + & + & + & + & + & + \\
\hline & $\mathrm{x}$ & Mytilaster minimus (Poli, 1795) & & + & + & + & + & + & + & + \\
\hline & $\mathrm{x}$ & Mytilus galloprovincialis Lamarck, 1819 & & + & + & + & + & + & + & + \\
\hline & & Musculus costulatus (Risso,1826) & & & & & + & + & + & \\
\hline PINNIDAE & & Pinna nobilis Linnaeus, 1758 & + & & & & & + & & \\
\hline Pectinidae & & Mimachlamys varia (Linnaeus, 1758) & & + & + & + & + & + & + & + \\
\hline ANOMIIDAE & & Anomia ephippium Linnaeus, 1758 & & & & & + & & & + \\
\hline OSTREIDAE & $\mathrm{x}$ & Crassostrea sp. & & + & + & + & + & + & + & + \\
\hline \multirow[t]{3}{*}{ LUCINIDAE } & & Loripinus fragilis (Philippi, 1836) & & & & & & & & + \\
\hline & & Ctena decussata (Costa O.G., 1829) & & & & & & & + & + \\
\hline & $\mathrm{x}$ & Loripes lucinalis (Lamarck, 1818) & + & + & & + & & + & & + \\
\hline Chamidae & & Chama gryphoides Linnaeus, 1758 & & & & & + & & + & \\
\hline LASAEIDAE & & Hemilepton nitidum (Turton, 1822) & + & + & + & + & + & & & \\
\hline \multirow[t]{3}{*}{ CARDIIDAE } & & Acanthocardia tuberculata (Linnaeus, 1758) & & & & & & & & + \\
\hline & $\mathrm{x}$ & $\begin{array}{l}\text { Cerastoderma spp. glaucum (Bruguière, 1789) complex } \\
\text { (see Appendix 2) }\end{array}$ & + & + & + & + & + & + & + & + \\
\hline & $\mathrm{x}$ & Parvicardium exiguum (Gmelin, 1791) & & & & & + & & & \\
\hline \multirow[t]{3}{*}{ MACTRIDAE } & & Eastonia rugosa (Helbling, 1779) & & & & & & + & + & + \\
\hline & & Lutraria oblonga (Gmelin, 1791) & & & & & & + & + & + \\
\hline & & $\begin{array}{l}\text { Mactra spp. stultorum (Linnaeus, 1758) complex } \\
\text { (see Guarniero et al. 2010) }\end{array}$ & & & & & & & & + \\
\hline SOLENIDAE & & Solen marginatus Pulteney, 1799 & & & & & & & & + \\
\hline Pharidae & & Ensis minor (Chenu, 1843) & & & & & & & & + \\
\hline \multirow[t]{6}{*}{ Tellinidae } & & Angulus incarnatus (Linnaeus, 1758) & & & & & & & & + \\
\hline & $\mathrm{x}$ & Angulus tenuis (da Costa, 1778 ) & & & & & & & & + \\
\hline & $\mathrm{x}$ & Gastrana fragilis (Linnaeus, 1758) & & & & & + & + & + & + \\
\hline & & Peronaea planata (Linnaeus, 1758) & & & & & + & + & + & + \\
\hline & & Psammotreta cumana (Costa O.G., 1829) & & & & & & + & + & + \\
\hline & & Tellina nitida Poli, 1791 & & & & & + & + & + & + \\
\hline \multirow[t]{2}{*}{ Donacidae } & & Donax semistriatus Poli, 1795 & & & & & & & & + \\
\hline & & Donax trunculus Linnaeus, 1758 & & & & & & & & + \\
\hline PSAMMOBIIDAE & & Gari depressa (Pennant, 1777) & & & & & & & + & + \\
\hline \multirow[t]{3}{*}{ SEMELIDAE } & & Abra alba (Wood W., 1802) & + & + & & + & + & & & \\
\hline & $\mathrm{x}$ & Abra segmentum (Récluz, 1843) & & & & & + & + & + & + \\
\hline & & Scrobicularia plana (da Costa, 1778) & + & + & & + & + & & & \\
\hline SOLECURTIDAE & & Solecurtus strigilatus (Linnaeus, 1758) & & & & & & & & + \\
\hline \multirow[t]{9}{*}{ VENERIDAE } & & Callista chione (Linnaeus, 1758) & & & & & & & & + \\
\hline & & Chamelea gallina (Linnaeus, 1758) & & & & & & & + & + \\
\hline & & Dosinia exoleta (Linnaeus, 1758) & & & & & & & + & + \\
\hline & & Petricola lithophaga (Retzius, 1788) & & & & & & & & + \\
\hline & $\mathrm{x}$ & Polititapes aureus (Gmelin, 1791) & + & + & & + & + & + & + & + \\
\hline & & Venerupis corrugata (Gmelin, 1791) & & & & & + & + & + & + \\
\hline & $\mathrm{x}$ & Venerupis decussata (Linnaeus, 1758) & + & + & & + & + & + & + & + \\
\hline & $\mathrm{x}$ & Venerupis philippinarum (Adams A. and Reeve, 1850) & + & + & & + & + & + & + & + \\
\hline & & Venus verrucosa Linnaeus, 1758 & & & & & + & + & + & + \\
\hline Pholadidae & & Barnea candida (Linnaeus, 1758) & & & & & & & + & + \\
\hline & & Pholas dactylus Linnaeus, 1758 & & & & & & & + & + \\
\hline CEPHALOPODA & & & & & & & & & & \\
\hline SEPIIDAE & $\mathrm{x}$ & Sepia officinalis Linnaeus, 1758 & & & & & + & + & + & + \\
\hline OCTOPODIDAE & & Callistoctopus macropus (Risso, 1826) & & & & & + & + & + & + \\
\hline & & Octopus vulgaris Cuvier, 1797 & & & & & + & + & + & + \\
\hline
\end{tabular}

1936 and Rissoa paulae Coen in Brunelli and Cannicci, 1940) (Fig. 2), and their new synonyms are proposed in Appendix 1 [as Mytilaster Monterosato, 1884, Mytilaster marioni (Locard, 1889) and Rissoa membranacea (J. Adams, 1800)]. M. marioni, Abra segmentum and Cerastoderma early colonized the whole basin, while Mytilus galloprovincialis showed a scattered distribution. The presence of marine species (Bittium reticulatum, Loripes lucinalis and Rissoa membranacea) was limited to the Bagnara arm and the Roman Channel area (Brunelli and Cannicci 1944, Ferrero 1961). Shellfish harvesting only started in 1955 (mussels: Ferrero 1961), while target species of professional fisheries were mainly commercial fishes.

\section{The 1966-1995 period}

Twenty-three taxa had been recorded by past authors (Table 2, Appendix 1), whether introduced or 


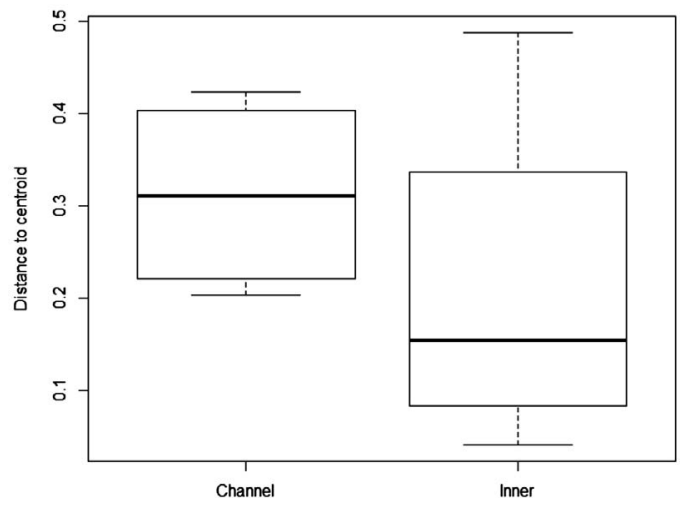

Fig. 4. Distance from the group centroid of the Channel and Inner Clusters of sites, using the Jaccard index as a dissimilarity measurement. The horizontal line within each box shows the median, the bottom and the tops of the box shows the 25 th and 75 th percentiles, respectively. The vertical dashed lines show either the maximum and minimum value or 1.5 times the interquartile range.

not. With the sole exception of Crassostrea angulata, locally introduced in 1966, the records hereby listed come from the 1974-1995 period. Rissoa membranacea disappeared from the area, though it had been widely recorded during the previous research, and Haminoea navicula was replaced by Haminoea orbignyana (Table 2, Appendix 1). Shellfish harvesting reached its maximum impact, with introductions or translocations in the field of several species (Perdicaro 1984, Mancini et al. 1988, Di Marco et al. 1990, Appendix 1). Among them, also the well-known Mediterranean native/alien competitors Ostrea edulis/Crassostrea angulata - $C$. gigas and Venerupis decussata/V. philippinarum, none of which naturally reached Sabaudia Lake.

\section{The 2005-2011 period}

Recent field studies covering Sabaudia Lake and first including the whole Roman Channel, recorded by census 119 taxa in 2005-2011, mostly belonging to Gastropoda (62 taxa) and Bivalvia (50 taxa). With the sole exception of Haminoea orbignyana, Heleobia stagnorum and Ecrobia ventrosa, the species recorded in 1974-1995 still continue living in the area (Tables 2, 3, Appendix 1).

The cluster analysis performed on the molluscan distributional pattern identified two groups of sites, Sabaudia Lake inner sites (S1-S4: Inner Cluster) and Roman Channel sites (S5-S8: Channel Cluster). The Inner Cluster had a mean number of $27.7 \pm 6.3$ species per site, significantly different from the $66.5 \pm 8.1$ species per site recorded in the Channel Cluster (sign test, $\mathrm{p}<0.001)$. In turn, the mean number of sites sharing the same species was $3.17 \pm 0.74$ for the Inner Cluster, significantly different from the average of $2.33 \pm 1.20$ sites sharing the same species in sites of the Channel Cluster (Wilcoxon test, $\mathrm{V}=930, \mathrm{p}<0.001$ ). The ordination of the clusters by non-metric multidimensional scaling is shown in Figure 3. It rendered a good fit of low stress (stress=0.02; Clarke and Warwick 1994). The Adonis multivariate test revealed significant differences in composition between the Inner and Channel Clusters $\left(\mathrm{F}_{1,6}=8.076, \mathrm{p}=0.042\right)$ (Table 4), although the results obtained in the multivariate dispersion of the groups were not significant (betadisper, $\mathrm{F}_{1,6}=0.854, \mathrm{p}=0.3911$ ) (Fig. 4). For the latter analysis, a higher distance from the group centroid is generally expected in areas with higher beta diversity (Anderson et al. 2006).

\section{DISCUSSION}

\section{Evolutionary states of the local molluscan biota}

According to literature data, museum material and field samplings, the Sabaudia Lake molluscan biota has gone through different evolutionary states, confirming with biological assessments the historical traced human driven shift from a freshwater to a strongly marine-influenced lagoon ecosystem. Records of subfossil taxa suggest that previous shifts to environmental features similar to those prevailing today have occurred in the

TABLE 4. - Summary of the commonest species observed in both clusters (MCSBC), the most frequently observed species exclusive to the Channel Cluster (MFSCC) and exclusive species of the Inner Cluster (ESIC), listed in systematic order within each cluster. Bold: alien species in the Italian territorial seawaters (Crocetta 2012, Crocetta et al. 2013).

\begin{tabular}{|c|c|c|}
\hline MCSBC & MFSCC & ESIC \\
\hline Gibbula adriatica (Philippi, 1844) & Chiton olivaceus Spengler, 1797 & Tectura virginea (Müller O.F., 1776) \\
\hline Gibbula albida (Gmelin, 1791) & Patella ulyssiponensis Gmelin, 1791 & Paludinella globularis (Hanley in Thorpe, 1844) \\
\hline Gibbula ardens (Von Salis, 1793) & Bolinus brandaris (Linnaeus, 1758) & Hydrobia acuta (Draparnaud, 1805) \\
\hline Hexaplex trunculus (Linnaeus, 1758) & Euthria cornea (Linnaeus, 1758) & Islamia pusilla (Piersanti, 1952) \\
\hline Stramonita haemastoma (Linnaeus, 1767) & Spurilla neapolitana (delle Chiaje, 1841) & Myosotella myosotis (Draparnaud, 1801) \\
\hline Cyclope neritea (Linnaeus, 1758) & Godiva quadricolor (Barnard, 1927) & \\
\hline Nassarius spp. corniculus (Olivi, 1792) complex & Arca noae Linnaeus, 1758 & \\
\hline Nassarius nitidus (Jeffreys, 1867) & Striarca lactea (Linnaeus, 1758) & \\
\hline Mytilaster marioni (Locard, 1889) & Gastrana fragilis (Linnaeus, 1758) & \\
\hline Mytilaster minimus (Poli, 1795) & Peronaeaplanata (Linnaeus, 1758) & \\
\hline Mytilus galloprovincialis Lamarck, 1819 & Tellina nitida Poli, 1791 & \\
\hline Mimachlamys varia (Linnaeus, 1758) & Abra segmentum (Récluz, 1843) & \\
\hline Crassostrea sp. & Venerupis corrugata (Gmelin, 1791) & \\
\hline Cerastoderma spp. glaucum (Bruguière, 1789) & Venus verrucosa Linnaeus, 1758 & \\
\hline complex Polititapes aureus (Gmelin, 1791) & Sepia officinalis Linnaeus, 1758 & \\
\hline Venerupis decussata (Linnaeus, 1758) & Callistoctopus macropus (Risso, 1826) & \\
\hline Venerupis philippinarum (Adams A. and Reeve, 1850) & Octopus vulgaris Cuvier, 1797 & \\
\hline
\end{tabular}


past, according to historical evidence, and represent the only known biological trace of the construction and re-opening of the Roman Channel during the Roman Empire and around 1700.

The opening of the two sea water connections (around 1930) and the continuous freshwater flowthrough into the lake have kept the salinity below $20 \%$ o during the investigated period (Brunelli and Cannicci, 1944, Ferrero 1961) and the analysis of the species recorded in 1933-1958, and of their spatial distribution, shows an early colonization of vacant niches by a few ecologically tolerant species.

About 40 years after its definitive geomorphologic conversion to a coastal lagoon, anthropogenic pressures on Sabaudia Lake peaked in the late 1970s. Sewage pollutants from the urban area were mainly discarded within the Annunziata arm, also leading to a high dystrophic crisis, and only since the 1980s has the area reached a steady state. Freshwater flow coming into the lake from drainage canals was, however, strongly reduced to meet agricultural needs, resulting in salinities higher than 30\%o (Perdicaro 1984). A progressive settlement in the area of marine molluscan species was noted (see species recorded during the 1966-1995 period), but the local introduction of several "native" Mediterranean species highlights intriguing questions regarding the strong uniformity in species composition of the molluscan fauna of the Mediterranean lagoon, and calls for the necessity of molecular analysis to elucidate the taxa distributional pattern not only on a global scale but also on a more regional one.

Finally, more than 100 species were recorded during the 2005-2011 period. Indeed, this may have been induced by the strongest searching effort ever achieved in the study area, as well as by the increasing taxonomical knowledge and the planned sampling design (covering all the different habitat types and first including the Roman Channel sea-lake connection). However, the data obtained in the present study confirm a definitive shift to a strongly marine-influenced lagoon ecosystem.

\section{Current molluscan assemblage: faunal and ecological insights}

The two clusters of sites identified in this study (Fig. 3 ) reflect a distribution pattern in agreement with the "confinement gradient" (Guelorget and Perthuisot 1984, 1992), which predicts a decreasing trend of species richness from less confined to inner lagoon zones. The Inner Cluster was mostly composed of a subset of species also observed in the Channel Cluster, except Paludinella globularis, Hydrobia acuta, Islamia pusilla, Myosotella myosotis and Tectura virginea (Tables 3, 4). Among them, species belonging to the Assimineidae and Hydrobiidae are well known as freshwater and estuarine inhabitants (Hershler and Frest 1996, Fukuda and Ponder 2003), and their presence only at S3 and S4 may be justified by a stronger freshwater flow that is absent at $\mathrm{S} 2$ and lower at S1 because of marine waters coming from Caterattino. Within the four internal sampling sites, the most represented families among gastropods were the herbivorous Trochidae and the carnivorous-necrofagous Muricidae and Nassaridae, whereas among bivalves they were Mytilidae and Veneridae (Table 3). Indeed, S2 and S4 share a high presence of bivalves (mainly Veneridae and Semelidae) because of the higher availability of a burrowing substratum, while S1 was partially separate from S2-S4 (Fig. 3). The scarce presence of hard substrata available for sessile (Crassostrea sp.) or partially sessile (Mytilidae, Mimachlamys varia) taxa, as well as of some herbivorous species, may be a possible explanation for this finding. In addition, the prevalent NW winds generate a fleeting current to the SE that is supposed to influence S1's ecological conditions, drifting marine water from the northern outfall to this spot: this is presumably at the base of the presence of a Pinna nobilis population in the area. Altogether, the low renewal of the marine element in the inner part influences environmental variables such as salinity, organic matter content, oxygen levels, temperature and grain size, which encompasses a restricted faunal composition (e.g. Gray 1974, Reizopoulou and Nicolaidou 2004, Basset et al. 2008, Magni et al. 2008).

Conversely, the inlet areas of the lagoon are influenced by both lake and sea water flows, and daily water exchanges within the Roman Channel imply that pelagic organisms, including molluscan larvae, constantly replenish the biotope. These environmental conditions favour a high species diversity (Carvalho et al. 2005, Koutsoubas et al. 2000), with molluscs often described as the most diverse faunal group in the sea inlet of marine lagoons (Millet and Guelorget 1994). This finding is in agreement with the high number of species observed within the Channel Cluster (S5-S8), considering that this very tiny spot $\left(0.27 \mathrm{~km}^{2}\right)$ hosted 113 taxa, and 82 were only recorded within it (Table 3). A molecular approach to the sampled species may clarify whether most of them occur as established populations (including some forms of resistance or resilience) or casual, but repetitive, occurrences. The S6 and S8 sites were indeed influenced by saline waters, as confirmed by their dominant macrofauna (Table 1), while S5 is a moderately marine-influenced zone, being a transitional zone between paralic and marine areas of Sabaudia Lake. Although 17 taxa were widely distributed in all four sampling sites (Table 3), differences among sampling sites were appreciable. Sites S6 and S7 shared the exclusive presence of some Rissoidae, Flabellina spp., Diodora graeca and Jorunna tomentosa, while S8 alone accounted for 70 taxa, and 15 were found only within it (Table 3). Among them, there are typical marine species such as Nassarius mutabilis, Acanthocardia tuberculata, Solen marginatus, Ensis minor, Donax semistriatus, Donax trunculus, Solecurtus strigilatus and Callista chione.

Finally, Terlizzi et al. (2009) showed that the use of presence-absence data is a valid approach for assessing 
beta diversity in molluscan assemblages. Nonetheless, our data show that this is not always possible between sites with clear differences in species richness. Results of the Wilcoxon test suggest a higher degree of homogeneity in species composition between sites at Sabaudia Lake. However, it seems that departures from homogeneity in species composition between sites within Sabaudia Lake created a wider range of multivariate dispersion than in the case of the Roman Channel. As such, sites from the Roman Channel showed a more homogeneous faunistic composition, while a higher range of dissimilarity between sites within Sabaudia Lake resulted in a higher distance from the group centroid (Fig. 4).

\section{Special emphasis on alien species}

Italian hotspots of introduction and secondary spreading of alien species were recently highlighted by the SIBM Allochthonous Species Group and Anna Occhipinti-Ambrogi (2010), who did not include any location in Latium. Our samplings showed that Venerupis philippinarum and Crassostrea specimens were among the most widespread species in the study area, the former being recorded at seven sites and the latter colonizing most of the available natural and artificial banks (Table 3). A wider study for the occurrence of Crassostrea oysters in the CNP marine area revealed its presence in the Circeo harbour (Latium, Tyrrhenian Sea) too, where it can be commonly found attached to the dock walls (Macali, unpublished data). Recent records of Haminoea japonica from Sabaudia Lake have considerably expanded its known Mediterranean range to the Tyrrhenian Sea (Crocetta et al. 2013) and, according to our field observations, this species has completely replaced populations of other native Haminoea species. This finding is paralleled by a similar observation by Hanson et al. (2013), who reported the complete replacement of the once common Haminoea vesicula (Gould, 1855) in Boundary Bay (North America). The records of Arcuatula senhousia confirm the presence of this species in the area (see Cossignani and Ardovini 2011). Its presence at S8 only (Fig. 1 and Table 3) would suggest a colonization of the area from the Roman Channel and not an introduction by mariculture. We are currently not aware of any further record from the Pontine Lagoons, but monitoring its local spread may be a future target. The presence of Godiva quadricolor and Bursatella leachii was first reported from Latium. Godiva quadricolor has only been reported in the Mediterranean Sea from the Gibraltar Strait area and Fusaro Lake (Campania, Tyrrhenian Sea) (Cervera et al. 2010), although its further presence in the Adriatic Sea (Piallassa Baiona, Ravenna) is also first reported herein (Rinaldi 2012 as Facelina auriculata). This would suggest that a correct assessment of its distribution may be mostly concealed by taxonomic impediments and the absence of field research. On the other hand, Bursatella leachii has been only recorded in the central Tyrrhenian Sea from Tus- cany, Sardinia and Campania (Crocetta et al. 2009). Finally, four individuals of the slipper limpet Crepidula fornicata were found attached to Crassostrea: this is the second record of this species in the CNP wetlands after its first record from Caprolace Lake (Bini 1983b). However, no established populations have been found in the area, so the species is conservatively considered as casual (Crocetta et al. 2013): these specimens may be the result of periodical re-introduction of Crassostrea oysters in the lake, presumably from the Atlantic shores. The same can be said for Rapana venosa: despite its recent collection in 2005 (Crocetta and Soppelsa 2006), the absence of further records suggests that its ephemeral introduction may be connected with mussel imports from the northern Adriatic Sea.

\section{ACKNOWLEDGEMENTS}

Henk Mienis (Israel) and Oz Rittner (Israel) provided photos of the studied type material. Marco Bodon (Italy), Juan Lucas Cervera (Spain), Ermanno Quaggiotto (Italy) and Ángel Valdés (California) shared their knowledge, respectively, on Hydrobia acuta and Islamia pusilla, on Anteaeolidiella lurana, on "Rissoa paulae" and on Haminoea japonica. Stefano Bartolini (Italy) provided the finding date of the record of "Cerastoderma glaucum" from Sabaudia. Joachim Langeneck (Italy) provided some requested bibliography. Stelios Katsanevakis (Italy), John Briggs (Oregon) and two anonymous referees gave suggestions on an early draft of the manuscript. We are grateful to all of them.

\section{REFERENCES}

Alia E., Russo G.F. 2003. Dinamica annuale del popolamento a Molluschi Bivalvi in stazioni a diverso grado di confinamento della laguna di Sabaudia (LT). Biologi Italiani 2: 34-39.

Anderson M.J. 2001. A new method for non-parametric multivariate analysis of variance. Austral Ecol. 26: 32-46.

Anderson M.J., Ellingsen K.E., McArdle B.H. 2006. Multivariate dispersion as a measure of beta diversity. Ecol. Lett. 9: 683-693.

André C., Lindegarth M., Jonsson P.R., Sundberg P. 1999. Species identification of bivalve larvae using random amplified polymorphic DNA (RAPD): differentiation between Cerastoderma edule and C. lamarcki. J. Mar. Biol. Assoc. U.K. 79: 563-565.

Barco A., Evans J., Schembri P.J., Taviani M., Oliverio M. 2013. Testing the applicability of DNA barcoding for Mediterranean species of top-shells (Gastropoda, Trochidae, Gibbula s.1.). Mar. Biol. Res. 9: 785-793.

Barco A., Houart R., Bonomolo G., Crocetta F., Oliverio M. in press. Molecular data reveal cryptic lineages within the northeastern Atlantic and Mediterranean small mussel drills of the Ocinebrina edwardsii complex (Mollusca: Gastropoda: Muricidae). Zool. J. Linnean Soc. doi: 10.1111/zoj.12069.

Basset A., Sabetta L., Sangiorgio F., Pinna M., Abbiati M., Ponti M., Fonda Umani S., Faresi L., Simboura N., Nicolaidou A., Arvanitidis C., Moncheva S., Trajanova A., Georgescu L., Beqiraj S., Orfanidis S., Reizopoulou S. 2008. Biodiversity conservation in Mediterranean and Black Sea lagoons: a trait-oriented approach to benthic invertebrate guilds. Aquat. Conserv. 18: 4-15.

Bini G. 1983a. Note sulla validità specifica di Haminoea orbignyana (Férussac, 1822) Mollusca - Opistobranchia. Studi per l'Ecologia del Quaternario 5: 127-129.

Bini G. 1983b. Immissione antropica di molluschi esotici nel Mediterraneo: I - Il lago di Caprolace. Studi per l'Ecologia del Quaternario 5: 113-125. 
Bini G. 1987. Studio della malacofauna vivente e subfossile del Lago di Paola in relazione alla salinità. Studi per l'Ecologia del Ouaternario 9: 113-125.

Borcard D., Gillet F., Legendre P. 2011. Numerical ecology with R. Springer, New York, $306 \mathrm{pp}$.

Boisselier-Dubayle M.C., Gofas S. 1999. Genetic relationships between marine and marginal-marine populations of Cerithium species from the Mediterranean Sea. Mar. Biol. 135: 671-682.

Beatley T. 1991. Protecting biodiversity in coastal environments: introduction and overview. Ocean Coast. Manage. 19: 1-19.

Brock V., Christiansen G. 1989. Evolution of Cardium (Cerastoderma) edule, C. lamarcki and C. glaucum: studies of DNAvariation. Mar. Biol. 102: 505-511.

Brunelli G., Cannicci G. 1934. Notizie preliminari sulle caratteristiche chimiche e biologiche del Lago di Sabaudia (Paola). Atti della Reale Accademia Nazionale dei Lincei 12: 345-351.

Brunelli G., Cannicci G. 1940. Il lago di Sabaudia (Paola). Boll. Pesca Piscic. Idrobiol. 16: 35-54.

Brunelli G., Cannicci G. 1944. Le caratteristiche biologiche del lago di Sabaudia. Atti della Accademia d'Italia. Memorie della Classe di Scienze Fisiche, Matematiche e Naturali 16: 663-731.

Carvalho S., Moura A., Gaspar M.B., Pereira P., Cancela da Fonseca L., Falcão M., Drago T., Leitão F., Regala J. 2005. Spatial and inter-annual variability of the macrobenthic communities within a coastal lagoon (Óbidos lagoon) and its relationship with environmental parameters. Acta Oecol. 27: 143-159.

Cervera J.L., Tamsouri N., Moukrim A., Villani G. 2010. New records of two alien opisthobranch mollusks from the northeastern Atlantic: Polycera hedgpethi and Godiva quadricolor. Mar. Biodiver. Rec. 3: e51.

Clarke K.R., Warwick R.M. 1994. Change in marine communities: an approach to statistical analysis and interpretation. Plymouth Marine Laboratory, Plymouth, 144 pp.

Claudet J., Fraschetti S. 2010. Human-driven impacts on marine habitats: A regional meta-analysis in the Mediterranean Sea Biol. Conserv. 143: 2195-2206.

Coen G. 1936. Mytilodonta nuovo genere di molluschi lamellibranchi. Acta Pontificia. Academia Scientiarum Novorum Lyncaeorum, Civitate Vaticana 88: 298-301.

Coll M., Piroddi C., Steenbeek J., Kaschner K., Lasram F.B.R., Aguzzi J., Ballesteros E., Bianchi C.N., Corbera J., Dailianis T., Danovaro R., Estrada M., Froglia C., Galil B., Gasol J.M., Gertwagen J., Gil J., Guilhaumon F., Kesner-Reyes K., Kitsos M.S., Koukouras A., Lampadariou N., Laxamana E., de la Cuadra C.M.L., Lotze H.K., Martin D., Mouillot D., Oro D., Raicevich S., Rius-Barile J., Saiz-Salinas J.I., San Vicente C., Somot S., Templado J., Turon X., Vafidis D., Villanueva R., Voultsiadou E. 2010. The Biodiversity of the Mediterranean Sea: Estimates, Patterns, and Threats. PLOS ONE 5: e11842.

Cossignani T., Ardovini R. 2011. Malacologia Mediterranea. Atlante delle conchiglie del Mediterraneo - 7.500 foto a colori. L'Informatore Piceno, Ancona, 540 pp.

Costello M.J., Philippe B., Emblow C.S., Legakis A. 2006. European marine biodiversity inventory and taxonomic resources: state of the art and gaps in knowledge. Mar. Ecol. Prog. Ser 316: $257-268$

Crawley M.J. 2007. The R book. Wiley, Chichester, 1076 pp.

Crocetta F., Soppelsa O. 2006. Primi ritrovamenti di Rapana venosa (Valenciennes, 1846) per alcune lagune costiere italiane. Atti del Museo Civico di Storia Naturale di Trieste 52: 215-218.

Crocetta F., Renda W., Vazzana A. 2009. Alien Mollusca along the Calabrian shores of the Messina Strait area and a review of their distribution in the Italian Seas. Boll. Malacol. 45: 15-30.

Crocetta F. 2012. Marine alien Mollusca in Italy: a critical review and state of the knowledge. J. Mar. Biol. Assoc. U.K. 92: $1357-1365$

Crocetta F., Macali A., Furfaro G., Cooke S., Villani G., Valdés Á. 2013. Alien molluscan species established along the Italian shores: an update, with discussions on some Mediterranean "alien species" categories. Zookeys 277: 91-108.

Davenport J., Davenport J.L. 2006. The impact of tourism and personal leisure transport on coastal environments: a review. Estuar. Coast. Shelf Sci. 67: 280-292.

Di Marco P., Lombardi F., Rambaldi E. 1990. Allevamento sperimentale della vongola verace Tapes philippinarum nel Lago di Sabaudia. Quad. Ist. Idrobiol. Aquacolt. Brunelli 10: 15-32.

Ferrero L. 1961. Ricerche fisico-chimiche e biologiche sui laghi sal- mastri pontini in relazione alla produttività - II. Il lago di Paola (Sabaudia). Boll. Pesca Piscic. Idrobiol. 16: 173-203.

Fukuda H., Ponder W.F. 2003. Australian freshwater assimineids, with a synopsis of the recent genus-group taxa of the Assimineidae (Mollusca: Caenogastropoda: Rissooidea). J. Nat. Hist. 37: $1977-2032$

Garilli V., Galletti L. 2006. Taxonomical characters for distinguishing Cerithium lividulum Risso, 1826, and C. renovatum Monterosato, 1884 (Gastropoda, Caenogastropoda, Cerithiidae). Basteria 70: 109-122.

Gofas S., Garilli V., Boisselier-Dubayle M.C. 2004. Nomenclature of the smaller Mediterraneum Cerithium species. Boll. Malacol. 39: 95-104.

Gray J.S. 1974. Animal-sediment relationships. Oceanogr. Mar. Biol. Ann. Rev. 12: 223-261.

Guarniero F., Plazzi A., Bonfitto A., Rinaldi M., Trentini M., Passamonti M. 2010. The bivalve mollusc Mactra corallina: genetic evidence of existing sibling species. J. Mar. Biol. Assoc. U. K. 90: 633-644

Guelorget O., Perthuisot J.P. 1984. Indicateurs biologiques et diagnose ècologique dans le domaine paralique. Bull. Ecol. 15: $67-76$.

Guelorget O., Perthuisot J.P. 1992. Paralic ecosystems. Biological organization and functioning. Vie Milieu 42: 215-225.

Hanson D., Hirano Y., Valdés A. 2013. Population genetics of Haminoea (Haloa) japonica Pilsbry, 1895, a widespread nonnative sea slug (Mollusca: Opisthobranchia) in North America and Europe. Biol. Invasions 15: 395-406.

Hendrickx M., Harvey A.W. 1999. Checklist of anomuran crabs (Crustacea: Decapoda) from the Eastern Tropical Pacific. Belg. J. Zool. 129: 363-389.

Hershler R., Frest T.J. 1996. A Review of the North American Freshwater Snail Genus Fluminicola (Hydrobiidae). Smithson. Contrib. Zool. 583: 1-41.

Hummel H., Wolowicz M., Bogaards R.H. 1994. Genetic variability and relationships for populations of Cerastoderma edule and of Cerastoderma glaucum complex. Neth. J. Sea Res. 33: 81-89.

Iannotta M.A., Toscano F., Patti F.P. 2008. Nassarius corniculus (Olivi, 1792) (Caenogastropoda: Nassariidae): a model of environmental complexity of Italian brackish and marine habitats. Mar. Ecol. 30: 106-115.

Koutsoubas D., Arvanitidis C., Dounas C., Drummond L. 2000. Community structure and dynamics of the Molluscan Fauna in a Mediterranean lagoon (Gialova lagoon, SW Greece). Belg. J. Zool. 130: 131-138

Lapègue S., Batista R.M., Heurtebise S., Yu Z.N., Boudry P. 2004. Evidence for the presence of the Portuguese oyster, Crassostrea angulata, in Northern China. J. Shell. Res. 23: 759-763.

Legendre P., Legendre L. 1998. Numerical ecology. Elsevier, Amsterdam, $853 \mathrm{pp}$.

Liu J., Li Q., Kong L., Yu H., Zheng X. 2011. Identifying the true oysters (Bivalvia: Ostreidae) with mitochondrial phylogeny and distance-based DNA barcoding. Mol. Ecol. Res. 11: 820-830.

Lloret J., Riera V. 2008. Evolution of a Mediterranean coastal zone: human impacts on the marine environment of Cape Creus. Environ. Manage. 42: 977-988.

López-Flores I., de la Herrán R., Garrido-Ramos M.A., Boudry P., Ruiz-Rejón C., Ruiz-Rejón M.2004. The molecular phylogeny of oysters based on a satellite DNA related to transposons. Gene 339: $181-188$

Magni P., Como S., Cucco A., De Falco G., Domenici P., Ghezzo M., Lefrançois C., Simeone S., Perilli A. 2008. A Multidisciplinary and Ecosystemic Approach in the Oristano Lagoon-Gulf System (Sardinia, Italy) as a Tool in Management Plans. Transitional Waters Bull. 2: 41-62.

Mancini L., Di Girolamo I., Pietrangeli B., Aulicino F.A., Volterra L., Pollaci V. 1988. Flora batterica di mitili stabulati nel Lago di Sabaudia. Quad. Ist. Idrobiol. Aquacolt. Brunelli 8: 29-36.

Mannino M.A., Thomas K.D. 2002. Depletion of a resource? The impact of prehistoric human foraging on intertidal mollusc communities and its significance for human settlement, mobility and dispersal. World Archaeol. 33: 452-474.

Matta F. 1968. Sull'acclimatazione e l'accrescimento di Gryphaea angulata Lmk. in alcune regioni italiane. Boll. Pesca Piscic. Idrobiol. 23: 171-258.

Mikkelsen P.M., Cracraft J. 2001. Marine biodiversity and the need for systematic inventories. Bull. Mar. Sci. 69: 525-534. 
Millet B., Guelorget O. 1994. Spatial and seasonal variability in the relationship between benthic communities and physical environment in a lagoon ecosystem. Mar. Ecol. Prog. Ser. 108: $161-174$.

Nixon S.W. 1995. Coastal marine eutrophication: a definition, social causes, and future concerns. Ophelia 41:199-219.

Occhipinti-Ambrogi A. 2007. Global change and marine communities: alien species and climate change. Mar. Pollut. Bull. 55: 342-352.

Oksanen J., Kindt R., Legendre P., O’Hara R.B. 2006. VEGAN: Community Ecology Package Version 1.8-2. Accessed at http:// cran.r-project.org.

Perdicaro R. 1983. Osservazioni sulle condizioni idrologiche del Lago di Sabaudia o di Paola nel biennio 1982-1983. Quad. Ist. Idrobiol. Aquacolt. Brunelli 3: 59-72.

Perdicaro R. 1984. Condizioni chimico-fisiche del Lago di Sabaudia o di Paola dal 1933 al 1984. Quad. Ist. Idrobiol. Aquacolt. Brunelli 4: 71-139.

R Development Core Team, 2009 R: a Language and Environment for Statistical Computing. R Foundation for Statistical Computing. Accessed at: http://www.R-project.org.

Reece K.S., Cordes J.F., Stubbs J.B., Hudson K.L., Francis E.A. 2008. Molecular phylogenies help resolve taxonomic confusion with Asian Crassostrea oyster species. Mar. Biol. 153: 709-721.

Reizopoulou S., Nicolaidou A. 2004. Benthic diversity of coastal brackish-water lagoons in western Greece. Aquat. Conserv. 14: 93-102.

Rinaldi A. 2012. Atlante della fauna e flora marina dell'Adriatico nord-occidentale. La Mandragola Editrice, Imola, 640 pp.

Rolán E., Luque A.A. 1994. Nassarius reticulatus (Linneus, 1758) y Nassarius nitidus (Jeffreys, 1867) (Gastropoda, Nassariidae), dos especies válidas de los mares de Europa. Iberus 12: 59-76.

Sá-Pinto A., Baird S.J.E., Pinho C., Alexandrino P., Branco M. 2010. A three-way contact zone between forms of Patella rustica (Mollusca: Patellidae) in the central Mediterranean Sea. Biol. J. Linn. Soc. 100: 154-169.

Scaperrotta M., Bartolini S., Bogi C. 2012. Accrescimenti. Vol IV. L'Informatore Piceno, Ancona, 185 pp.

S.I.B.M. Allochthonous Species Group, Occhipinti-Ambrogi A. 2010. Hotspots of introduction of marine alien species in Italian Seas. Rapp. Comm. Int. Mer Médit. 39: 427.

Tarnowska K., Krakau M., Jacobsen S., Wolowicz M., Féral J.P., Chenuil A. 2012. Comparative phylogeography of two sister (congeneric) species of cardiid bivalve: strong influence of habitat, life history and post-glacial history. Estuar. Coast. Shelf Sci. 107: 150-158.

Terlizzi A., Anderson M.J., Bevilacqua S., Fraschetti S., Wlodarska-Kowalczuk M., Ellingsen K.E. 2009. Beta diversity and taxonomic sufficiency: do higher-level taxa reflect heterogeneity in species composition? Diversity Distrib. 15: 450-458.

WoRMS, 2012 World Register of Marine Species - http://www. marinespecies.org.

Scient. ed.: J. Templado.

Received March 27, 2013. Accepted September 5, 2013.

Published online October 3, 2013.

APPENDIX 1. - Molluscan taxa cited from Sabaudia Lake (as * live; + subfossil), with remarks, and taxonomical accounts on taxa described with Sabaudia Lake as type locality. The use (or not) of parentheses for taxon authorships has been reported as employed in each literature record. The original style (caps v. small, italics v. roman) has been corrected, while the original spelling or stated authority has been maintained and is followed by [sic!] if wrong. Abbreviations used: HUJ, The Hebrew University of Jerusalem; \# the taxonomic status of Crassostrea gigas and C. angulata is still controversial, with some authors considering them as different species (e.g. Lapègue et al. 2004, Liu et al. 2011) and others regarding them as conspecific (e.g. López-Flores et al. 2004, Reece et al. 2008). They are here conservatively treated as different species.

Family Trochidae Rafinesque, 1815

Gibbula adansonii (Payraudeau, 1826) complex

Literature records. Gibbula (Colliculus) adansonii (Payraudeau, 1826) - Bini 1987: 113 (+).

Family CerithiIdae Fleming, 1822

Bittium reticulatum (da Costa, 1778)

Literature records. Bittium reticulatum B., D., D. [sic!] var. paludosa - Brunelli and Cannicci 1934: 349 (*), Brunelli and Cannicci 1940: 46 (*), Brunelli and Cannicci 1944: $680(*)$; Bittium reticulatum Da Costa [sic!] - Ferrero 1961: 185 (*); Bittium reticulatum paludosum B.D.D., 1884 - Bini 1987: 114 (+, *).

\section{Cerithium sp.}

Literature records. Cerithium sp. - Ferrero 1961: 187 (+). Cerithium rupestre Risso, 1826 - Bini 1987: $114(+)$.

Remarks. Early Mediterranean records of Cerithium rupestre are to be referred to both $C$. lividulum Risso, 1826 and $C$. renovatum Monterosato, 1884, as the type material of $C$. rupestre Risso, 1826 belongs to a species of the Cerithium spp. vulgatum Bruguière, 1792 complex (Gofas et al. 2004, Garilli and Galletti 2006), and therefore we cannot taxonomically adjust it with confidence.
Cerithium spp. vulgatum Bruguière, 1792 complex

Literature records. Cerithium vulgatum (Bruguière, 1792) [sic!] - Bini 1987: 115 (+).

Family Cochliopidae Tryon, 1866

Heleobia stagnorum (Gmelin, 1791)

Literature records. Heleobia stagnorum (Gmelin, 1791) complex! - Bini 1987: 114 (*).

Family Rissoidae Gray, 1847

Pusillina cf. lineolata (Michaud, 1832)

Literature records. Turboella cfr. lineolata (Michaud, 1832) - Bini 1987: 114 (+).

Rissoa membranacea (Adams J., 1800)

Literature records. Rissoa sabaudiae Coen, n. sp. - Brunelli and Cannicci, 1934: 349 (*), Brunelli and Cannicci, 1940: 46 (*), Brunelli and Cannicci, 1944: 680 (*); Rissoa (Rissostomia) paulae n. sp. - Coen in Brunelli and Cannicci, 1940: 46-47 (*); Rissoa (Rissostomia) paulae n. sp. - Coen in Brunelli and Cannicci, 1944: 680-681, Fig. 8 (*); Rissoa sp. - Ferrero 1961: $185(*)$.

Remarks. Rissoa sabaudiae is nomen nudum (ICZN, 1999: Art. 12). Rissoa paulae Coen in Brunelli and Cannicci, 1940 (Fig. 2F-H) is here considered a junior 
synonym of Rissoa membranacea (Adams J., 1800) on the basis of the analyses of the type material (Coen 9652 - HUJ 53487) and of specimens stored in the private collection of the authors and belonging to this taxon. The taxon listed as "Rissoa sp." by Ferrero (1961) is presumably the Rissoa paulae described by Coen.

Family HydrobiIdae Stimpson, 1865

Ecrobia ventrosa (Montagu, 1803)

Literature records. Ventrosia ventrosa (Montagu, 1803) complex - Bini 1987: 114 (*).

Hydrobia acuta (Draparnaud, 1805)

Literature records. Hydrobia acuta (Drpd.) - Ferrero 1961: 185 (*); Hydrobia acuta (Draparnaud, 1805) complex - Bini 1987: 114 (*).

\section{Hydrobia sp.}

Literature records. Hydrobia sp. - Brunelli and Cannicci 1934: 349 (*), Brunelli and Cannicci 1940: $46(*)$, Brunelli and Cannicci 1944: 680 (*), Ferrero 1961: $185(*)$.

Family Muricidae Rafinesque, 1815

Bolinus brandaris (Linnaeus, 1758)

Literature records. Bolinus brandaris (Linnaeus, 1758) - Bini 1987: 115, Fig. 1A (+).

Hexaplex trunculus (Linnaeus, 1758)

Literature records. Phyllonotus trunculus (Linnaeus, 1758) - Bini 1987: 115-116, Fig. 2 (+).

Rapana venosa (Valenciennes, 1846)

Literature records. Rapana venosa (Valenciennes, 1846) - Crocetta and Soppelsa 2006: 215-218 (*).

Family NASSARIIDAE Iredale, 1916

Cyclope neritea (Linnaeus, 1758)

Literature records. Cyclope neritea (Linnaeus, 1758) - Bini 1987: 116, Fig. 3A, B (+, *).

Nassarius spp. corniculum (Olivi, 1792) complex

Literature records. Nassarius (Gussonea) corniculum (Olivi, 1792) - Bini 1987: 117, Fig. 5A (+, *).

Nassarius nitidus (Jeffreys, 1867)

Literature records. Hinia reticulata (Linnaeus, 1758) - Bini 1987: 116-117, Fig. 4B (+).

Remarks. Early central Mediterranean records of Hinia reticulata in lagoons are to be referred to Nassarius nitidus (Jeffreys, 1867) as the type material of Buccinum reticulatum Linnaeus, 1758 belongs to a different species (Rolán and Luque 1994).

Family BULlidAe Gray, 1827

Bulla striata Bruguière, 1792

Literature records. Bulla striata Bruguière, 1789 [sic!] - Bini 1987: 117-118 (+).
Family Haminoeidae Pilsbry, 1895

Haminoea navicula (da Costa, 1778)

Literature records. Haminea navicula (D. C.) [sic!] - Ferrero 1961: $185\left(^{*}\right)$.

Haminoea orbignyana (Férussac, 1822)

Literature records. Haminoea orbignyana (Férussac, 1822) - Bini 1983: 127-129, Fig. 1 (*); Bini 1987: $118(*)$.

Family Mytilidae Rafinesque, 1815

Arcuatula senhousia (Benson in Cantor, 1842)

Literature records. Musculista senhousia - Cossigniani and Ardovini 2011: 410, Figure (*).

Mytilaster marioni (Locard, 1889)

Literature records. Mytilodonta, gen. nov. - Coen, 1936: 298-301, figs. 1-8 (*); Mytilodonta Coen, nov. gen. - Brunelli and Cannicci, 1940: 46 (*), Brunelli and Cannicci, 1944: 680 (*); Mytilodonta paulae, nov. spec. - Coen, 1936: 298-301, figs. 1-8 (*); Mytilodonta paulae Coen, nov. spec. - Brunelli and Cannicci, 1940 : 46 (*), Brunelli and Cannicci, 1944: 680 (*); Mytilidonta [sic!] paulae, n. sp. - Coen in Brunelli and Cannicci, 1940: $47(*)$; Coen in Brunelli and Cannicci, 1944: 681-682, figs. 9-10 (*); Brachydontes marioni (Loc.) - Ferrero 1961: $185(*)$; Mytilaster marioni Alia and Russo 2003: 34-39 (*).

Remarks. Mytilodonta Coen, 1936 and Mytilodonta paulae Coen, 1936 (Fig. 2A-E) are respectively considered junior synonyms of the genus Mytilaster Monterosato, 1884 and of the taxon Mytilaster marioni (Locard, 1889) on the basis of the analyses of the original description and the comparison between the type material (Coen 10634a and Coen 10634b HUJ 53488 and HUJ 53489) and the material hereby sampled, enriched by Mediterranean Mytilaster spp. stored in the private collection of the authors. Teeth of different strength (sometimes very weak) are often present in the ligament area of the Mediterranean $M y$ tilaster species, but can be covered by the ligament itself, thus suggesting their absence: this may have induced Coen to establish a new genus and a new species.

\section{Mytilaster minimus (Poli, 1795)}

Literature records. Mytilaster minimus - Coen 1936: 299 (*); Mytilaster minimus (Poli, 1795) - Bini 1987: 118-119, Fig. $6(*)$.

Mytilus galloprovincialis Lamarck, 1819

Literature records. Mytilus galloprovincialis Locard [sic!] - Brunelli and Cannicci 1934: 349 (*), Brunelli and Cannicci 1940: 46 (*), Brunelli and Cannicci 1944: 680 (*); Mytilus galloprovincialis Lamk - Ferrero 1961: 185 (*); Mytilus - Perdicaro 1984: 75 (*); Mytilus galloprovincialis Lamarck, 1819 - Bini 1987: 119 (+); Mytilus galloprovincialis - Mancini et al. 1988: 29-36(*). 
Remarks. Mancini et al. (1988) reported translocations and/or temporary introductions (before marketing) of specimens coming from other Italian localities (Gaeta, Goro and Manfredonia).

Family Ostreidae Rafinesque, 1815

\# Crassostrea angulata (Lamarck, 1819)

Literature records. Gryphaea angulata Lmk. Matta 1968: 232-238 (*).

Remarks. Temporary introduced in 1966 as a suspended culture (Matta 1968).

\# Crassostrea gigas (Thunberg, 1793)

Literature records. Crassostrea gigas - Perdicaro 1984: $75(*)$.

Remarks. Locally introduced in the field in the 80's (Perdicaro 1984).

Ostrea edulis Linnaeus, 1758

Literature records. Ostrea edulis - Perdicaro 1984: $75(*)$.

Remarks. Locally introduced in the 80's (Perdicaro 1984).

Family LUCINIDAE Fleming, 1828

Loripes lucinalis (Lamarck, 1818)

Literature records. Loripes lacteus L. var. squamosa - Brunelli and Cannicci 1934: 349 (*), Brunelli and Cannicci 1940: 46 (*), Brunelli and Cannicci 1944: 680 (*); Loripes lacteus L. [sic!] - Ferrero 1961: 185 (*); Loripes lacteus (Linnaeus, 1758) - Bini 1978: 119-120 (+); Loripes lacteus - Alia and Russo 2003: 34-39 (*).

Remarks. Records of Loripes lacteus are to be referred to Loripes lucinalis (Lamarck, 1818) as the type material of Tellina lactea Linnaeus, 1758 belong to Mysia undata (Pennant, 1777) (S. Gofas, unpublished data).

\section{Family CARDIIDAE Lamarck, 1809 Cerastoderma spp. glaucum (Bruguière, 1789) complex}

Literature records. Cerastoderma lamarkii [sic!] Reeve var. lacunaris - Brunelli and Cannicci 1934: 349 (*), Brunelli and Cannicci 1940: 46 (*), Brunelli and Cannicci 1944: 680 (*); Cerastoderma lamarkii [sic!] Reeve var. flabellata - Brunelli and Cannicci 1934: 349 (*), Brunelli and Cannicci 1940: 46 (*), Brunelli and Cannicci 1944: $680(*)$; Cerastoderma reclidens [sic!] Coen - Brunelli and Cannicci 1934: 349 (*), Brunelli and Cannicci 1940: 46 (*), Brunelli and Cannicci 1944: 680 (*); Cardium glaucum Brug. [sic!] - Ferrero 1961: 185 (*); Cardium edule - Perdicaro 1984: 75 (*); Cerastoderma glaucum (Poiret [sic!], 1789) - Bini 1987: 121 (+, *); Scaperrotta et al. 2012: 140 (*); Cerastoderma glaucum - Alia and Russo 2003: 34-39 (*).

Remarks. Though several papers focusing on the genus Cerastoderma in the Atlanto-Mediterranean area offer different opinions (Brock and Christiansen 1989, Hummel et al. 1994, André et al. 1999, Tarnowska et al. 2012), the definitive taxonomy of the genus and the correct identification of the Mediterranean specimens are far from being elucidated, so herein we prefer to keep the literature records under "Cerastoderma spp. glaucum (Bruguière, 1789) complex".

The specimens from Sabaudia Lake figured by Scaperrotta et al. (2012) were found in 2011 (S. Bartolini, pers. comm.).

Parvicardium exiguum (Gmelin, 1791)

Literature records. Parvicardium exiguum - Alia and Russo 2003: 34-39 (*).

Family Tellinidae Blainville, 1814

Angulus tenuis (da Costa, 1778)

Literature records. Tellina (Angulus) tenuis Da [sic!] Costa, 1778 - Bini 1987: 120 (*).

Gastrana fragilis (Linnaeus, 1758)

Literature records. Gastrana fragilis - Alia and Russo 2003: 34-39 (*).

Family Semelidae Stoliczka, 1870

Abra segmentum (Récluz, 1843)

Literature records. Syndesmia ovata Philippi [sic!] - Brunelli and Cannicci 1934: 349 (*), Brunelli and Cannicci 1940: 46 (*), Brunelli and Cannicci 1944: 680 (*); Abra ovata (Phil.) - Ferrero 1961: 185 (*); Abra segmentum - Alia and Russo 2003: 34-39 (*).

Family VenERIDAe Rafinesque, 1815

Polititapes aureus (Gmelin, 1791)

Literature records. Venerupis aurea (Gmelin in L., 1791) - Bini 1987: $121(+, *)$; Paphia aurea - Alia and Russo 2003: 34-39 (*).

Venerupis decussata (Linnaeus, 1758)

Literature records. Venerupis decussata - Perdicaro 1984: 75 (*); Tapes (Ruditapes) decussatus (Linnaeus, 1758) - Bini 1987: $120(+, *)$; Tapes decussatus - Alia and Russo 2003: 34-39 (*).

Remarks. Locally introduced in the 60's (Perdicaro 1984).

Venerupis philippinarum (Adams A. and Reeve, 1850)

Literature records. Tapes philippinarum (Adams and Reeve, 1850) - Di Marco et al. 1990: 15-32 (*).

Remarks. Locally introduced in 1989 (Di Marco et al. 1990).

Family SEPIIDAE Leach, 1817

Sepia officinalis Linnaeus, 1758

Literature records. Sepia officinalis - Perdicaro 1984: $75(*)$.

Remarks. Perdicaro (1984) reported local introductions held in the 80's. 